\title{
Existence of Cross Product and Application
}

Krishna Kumar Chaudhary ${ }^{1}$, Bharat Sharma ${ }^{1}$, Raju Ram Thapa ${ }^{2}$, Chetan Sudedi ${ }^{1}$, Dr. Anjay Kumar Mishra ${ }^{1 *}$

${ }^{1}$ School of Engineering, Madan Bhandari Memorial Academy Nepal, Urlabari 3, Morang, Nepal

${ }^{2}$ Department of Mathematics, PG Campus Biratnagar, Nepal

DOI: $10.36347 /$ sjpms.2020.v07i10.001

| Received: 13.09.2020 | Accepted: 21.09.2020 | Published: 07.10.2020

*Corresponding author: Anjay Kumar Mishra

\section{Abstract}

\section{Original Research Article}

The paper aims to assess the existence of cross product in 3 dimensional, 7 dimensional and further explore the reasons for non existence of cross product does in other dimensions. The major focus of the research is on existence of cross product in $\mathrm{n}$ - dimension through casual and descriptive research design. Aside from this, applications of cross product in engineering mathematics, physics and computer science has been highlighted for empirical solution to several problems.

Keywords: 3 dimensional, 7 dimensional, $\mathrm{n}$ dimensional, cross product, application.

Copyright @ 2020: This is an open-access article distributed under the terms of the Creative Commons Attribution license which permits unrestricted use, distribution, and reproduction in any medium for non-commercial use (NonCommercial, or CC-BY-NC) provided the original author and source are credited.

\section{INTRODUCTION}

The vector product has gained attention of scholars from different fields. In this respect casual and descriptive research will highlight the application for empirical solution to multidisciplinary problems. The main goal of this article is to show when, or for which $\mathrm{n}$ there is a vector product on $\mathrm{R}^{\mathrm{n}}$. Except on $\mathrm{R}^{3}$, it is less known that the vector product $R^{0}, R^{1}$ and $R^{7}$. In this article we will provide constructive proof of this result and a similar approach used in a 1967 article in The American Mathematical Monthly. In general the first to prove the vector product exists on $\mathrm{R}^{\mathrm{n}}$, only if $\mathrm{n}=0,1,3$ and 7, was is Beno Eckmann, using an algebraic topology, he proved the claim with his weaker one of the vector product, i.e. requiring product continuity, rather than bilinearity. Later, this result was extended to non degenerate symmetric bilinear forms over characteristic fields other than two, and a close relationship was established vector product on $\mathrm{R}^{\mathrm{n}}$.

\section{RESEARCH METHODOLGY \\ Vector Product of $\mathbf{R}^{3}$}

Before answering the question about existence of a vector product on $\mathrm{R}^{\mathrm{n}}$. let's define a vector product on $\mathrm{R}^{3}$, and state its properties. The standard scalar product on $\mathrm{R}^{\mathrm{n}}$ is mapping: $\mathrm{R}^{\mathrm{n}} \times \mathrm{R}^{\mathrm{n}} \rightarrow \mathrm{R}$, defined by the following formula:

$$
\mathrm{x} . \mathrm{y}=\sum_{i=1}^{n} x_{i} y_{i}, \mathrm{x}=\left(\mathrm{x}_{1}, \mathrm{x}_{2}, \ldots \ldots, \mathrm{x}_{\mathrm{n}}\right), \mathrm{y}=\left(\mathrm{y}_{1}, \mathrm{y}_{2}, \ldots \ldots, \mathrm{y}_{\mathrm{n}}\right)
$$

Let us state the properties of the scalar product on $\mathrm{R}^{\mathrm{n}}$
(i) $\mathrm{x} . \mathrm{x} \geq 0, \mathrm{x} \in \mathrm{R}^{\mathrm{n}}$
(ii) $\mathrm{x} . \mathrm{x}=0 \Leftrightarrow \mathrm{x}=0, \mathrm{x} \in \mathrm{R}^{\mathrm{n}}$;
(iii) $(\alpha \mathrm{x}) \cdot \mathrm{y}=\alpha(\mathrm{x} \cdot \mathrm{y}), \alpha \in \mathrm{R} \quad \mathrm{x}, \mathrm{y} \in \mathrm{R}^{\mathrm{n}}$
(iv) $(x+y) \cdot z=x \cdot z+y \cdot z, x, y, z \in R^{n}$
(v) $x \cdot y=y \cdot x \quad x, y \in R^{n}$

From properties (iii), (iv) and (v) follows the linearilty of the scalar product in the second variable. We conclude that the scalar product on $\mathrm{R}^{\mathrm{n}}$ is symmetric, bilinear mapping. For vectors $x, y \in R^{n}$ we say that they are vertical or orthogonal, in the notation $\mathrm{x} \perp \mathrm{y}$, if $\mathrm{x} . \mathrm{y}=0$ Let us also define the norm on the vector space $\mathrm{R}^{\mathrm{n}}$.
The standard (Euclidean) norm on the vector space $\mathrm{R}^{\mathrm{n}}$ is the function $\|\cdot\|: R^{n} \rightarrow R, \mathrm{x} \in \mathrm{R}^{\mathrm{n}}$ is valid:

$$
\|x\|=\sqrt{x \cdot x}=\sqrt{\sum_{i=1}^{n} x_{i}^{2}}
$$

For the norm at $\mathrm{R}^{\mathrm{n}}$, it holds

(i) $\|x\| \geq 0, x \in R^{n}$;

(ii) $\|x\|=0$ if and only if $x=0$;

(iii) $\|\alpha x\|=|\alpha|\|x\|, \alpha \in R, x \in R^{n}$

(iv) $\|x+y\| \leq\|x\|+\|y\| \quad \mathrm{x}, \mathrm{y} \in R^{n}$ 
Thus, in addition to the standard norm defned above, $\mathrm{R}^{\mathrm{n}}$ is normalized space. For the vector $x \in R^{n}$ we say that it is unique or normalized if $\|x\|=1$

That is equivalent to $\mathrm{x} . \mathrm{X}=1$

In the case of vector space $\mathrm{R}^{\mathrm{n}}$, the norm is actually the length of the vector, so we use the notion $|x|$, instead of $\|x\|$. Then it is

$$
\mathrm{x.y}=|x||y| \operatorname{Cos} \theta
$$

$$
a \times b=\left|\begin{array}{lll}
e_{1} & e_{2} & e_{3} \\
\mathrm{a}_{1} & \mathrm{a}_{2} & \mathrm{a}_{3} \\
b_{1} & b_{2} & b_{3}
\end{array}\right|=\left(a_{2} b_{3}-a_{3} b_{2}\right) e_{1}-\left(a_{1} b_{3}-a_{3} b_{1}\right) e_{2}+\left(a_{1} b_{2}-a_{2} b_{1}\right) e_{3}
$$

Where the set $\left\{\mathrm{e}_{1}, \mathrm{e}_{2}, \mathrm{e}_{3}\right\}$ is the canonical base for $\mathrm{R}^{3}$

\section{Note 1.2}

For the vectors $\mathrm{e}_{1}, \mathrm{e}_{2}$ and $\mathrm{e}_{3}$ the following holds:

$$
e_{1} \times e_{2}=e_{3}, \quad e_{2} \times e_{3}=e_{1}, \quad e_{1} \times e_{3}=-e_{2}
$$

From definition 1.1 we see that, unlike a scalar product, a vector product of two vectors is

vector, not a scalar. Take two vectors $a, b \in \mathrm{R}^{3}$ and let $\mathrm{R}^{3}$ $\exists \mathrm{c}=a \times b$ their vector product. Thus, a vector that has its own:

(1) Length; the length of the vector $\mathrm{c}$ is equal to the area of the parallelogram determined by the vectors $\mathrm{a}$ and $\mathrm{b}$

$|c|=|a \times b|=|a||b| \sin \theta$

Where $\theta$ is the angle between the vectors a and b

(2) Direction; the vector product of the two vectors is perpendicular to both vectors. In our example $c$ is perpendicular to $a$ and $b$, i.e. to the plane in which $\mathrm{a}$ and $\mathrm{b}$ are located. Using scalar product, this can be written as:

c. $a=0$ and c. $b=0$

(3) Orientation; orientation vector $\mathrm{c}$ is determined by the right-handed rule. More precisely, by setting index finger in the direction of vector a and middle finger in the direction of vector $b$, orientation of vector $\mathrm{c}$ will show us a thumb. Replacement of vector order when calculating vector of the product results in the opposite orientation of the product, which tells us that it is vector anti commutative product.

Let us now state the properties of the vector product.

\section{Theorem 1.3}

Let a, b, c and $\mathrm{d} \in R^{3}, \alpha, \beta, \gamma$ and $\delta \in R$. Then it holds:

(i) $a \cdot(a \times b)=0$ and $b \cdot(a \times b)=0$, (verticality)

(ii) $(a \times b) \cdot(a \times b)+(a \cdot b)^{2}=(a \cdot a)(b \cdot b)$, (Pythagorean property)

(iii) $(\alpha \mathrm{a}+\beta \mathrm{b}) \times(\gamma \mathrm{c}+\delta \mathrm{d})=\alpha \gamma(a \times c)+$ $\alpha \delta(a \times d)+\beta \gamma(b \times c)+\beta \delta(b \times d)$. (Bilinearity)

We have already listed property (i) in the definition of a vector product, and we will call it verticality of the vector product, and the property (iii)
Where $\theta$ is the angle between the vectors $\mathrm{x}$ and $\mathrm{y}$

Let us now define the vector product on the vector space $\mathrm{R}^{3}$

\section{Definition 1.1}

The vector product is the binary operation $\mathrm{x}$ : $R^{3} \times R^{3} \rightarrow R^{3}$. For vectors $a=\left(a_{1}, a_{2}, a_{3}\right)$ and $b=\left(b_{1}, b_{2}, b_{3}\right)$ from $\mathrm{R}^{3}$ we define their vector product in the following way:

the bilinearity of the vector product. Notice that the property (ii) derives from:

$$
\begin{aligned}
& a \cdot b=|a||b| \cos \theta \text { and } \\
& |a \times b|=|a||b| \sin \theta
\end{aligned}
$$

And we shall call it the property of Pythagoras. All three properties are easily proved using a definition vector product at $R^{3}$. The vector product at $R^{3}$ is also anti commutative i.e. for each $\mathrm{a}, \mathrm{b} \in R^{3}$ is valid: $a \times b=-b \times a$, what pulls $a \times a=0$

Note that this property can be derived from properties (i), (ii) and (iii) from Theorem 1.3. we will analyze this further more.

\section{Vector Product of $\mathbf{R}^{\mathbf{n}}$}

In the previous, we defined the vector product on $\mathrm{R}^{3}$, and listed its properties. Now the goal is to show whether there exists, and if so, what the vector product looks like on $\mathrm{R}^{\mathrm{n}}$, for $\mathrm{n}>3$. In fact, we want to extend the vector product to $\mathrm{R}^{\mathrm{n}}$ so that it retains the properties of (i), (ii) and (iii) of the theorem 1.3. So we are interested in how to define such a function, if it is that at all possible. At $\mathrm{R}^{3}$, it is defined by the determinant of a $3 \times 3$ matrix. However, can we extend the vector product using the determinant? Let's see what our determinant in $\mathrm{R}^{4}$ would look like

$$
\left|\begin{array}{llll}
e_{1} & e_{2} & e_{3} & e_{4} \\
\mathrm{a}_{1} & \mathrm{a}_{2} & \mathrm{a}_{3} & \mathrm{a}_{4} \\
b_{1} & b_{2} & b_{3} & b_{4} \\
c_{1} & c_{2} & c_{3} & c_{4}
\end{array}\right|
$$

Although, for example, the perpendicular property is valid, a vector product defined in this way is not binary operation, because the third vector in the matrix also appeared. Because the determinant is a function over square matrices, this problem is difficult to avoid $\mathrm{R}^{\mathrm{n}}$, for $\mathrm{n}>3$.

Although perhaps surprisingly, a vector product with properties of verticality, bilinearity and Pythogorean property except for $\mathrm{R}^{3}$ can be defined on $\mathrm{R}^{\mathrm{n}}$ only for 0,1 and 7 . The idea of proving that result is to show that if for some $\mathrm{n}$ there exists a vector product on $\mathrm{R}^{\mathrm{n}}$, then we can find an orthonormal base $\left\{\mathrm{e}_{1}, \mathrm{e}_{2}\right.$, $\left.\ldots . . e_{n}\right\}$ such that for $\mathrm{i} \neq \mathrm{j}$ there exits a $\mathrm{k}$ for which 
$e_{i} \times e_{j}=a e_{k}$ where $\mathrm{a}=1$ or -1 . Where does the idea come from?

Namely, to define a vector product on $\mathrm{R}^{3}$ with all required properties, due to bilinearity is actually sufficient to define a vector multiplication table for ortho vectors standardized base. From Note 1.2 and the anti commutativity of the vector product we see how that table looks at $\mathrm{R}^{3}$

\begin{tabular}{|r|r|r|r|}
\hline$\times$ & $e_{1}$ & $e_{2}$ & $e_{3}$ \\
\hline$e_{1}$ & 0 & $e_{3}$ & $-e_{2}$ \\
\hline$e_{2}$ & $-e_{3}$ & 0 & $e_{1}$ \\
\hline$e_{3}$ & $e_{2}$ & $-e_{1}$ & 0 \\
\hline
\end{tabular}

Using the table and bilinearity, we can reconstruct the vector product of two arbitrary vectors $a=\left(a_{1}, a_{2}, a_{3}\right)$ and $b=\left(b_{1}, b_{2}, b_{3}\right)$

$$
\begin{aligned}
& a \times b=\left(a_{1} e_{1}+a_{2} e_{2}+a_{3} e_{3}\right) \times\left(b_{1} e_{1}+b_{2} e_{2}+b_{3} e_{3}\right) \\
& =a_{1} b_{1}\left(e_{1} \times e_{1}\right)+a_{1} b_{2}\left(e_{1} \times e_{2}\right)+a_{1} b_{3}\left(e_{1} \times e_{3}\right)+a_{2} b_{1}\left(e_{2} \times e_{1}\right)+a_{2} b_{2}\left(e_{2} \times e_{2}\right)+a_{2} b_{3}\left(e_{2} \times e_{3}\right) \\
& +a_{3} b_{1}\left(e_{3} \times e_{1}\right)+a_{3} b_{2}\left(e_{3} \times e_{2}\right)+a_{3} b_{3}\left(e_{3} \times e_{3}\right) \\
& =a_{1} b_{2} e_{3}-a_{1} b_{3} e_{2}-a_{2} b_{1} e_{3}+a_{2} b_{3} e_{1}+a_{3} b_{1} e_{2}-a_{3} b_{2} e_{1} \\
& =\left(a_{2} b_{3}-a_{3} b_{2}\right) e_{1}+\left(a_{3} b_{1}-a_{1} b_{3}\right) e_{2}+\left(a_{1} b_{2}-a_{2} b_{1}\right) e_{3} \\
& =\left|\begin{array}{lll}
e_{1} & e_{2} & e_{3} \\
\mathrm{a}_{1} & \mathrm{a}_{2} & \mathrm{a}_{3} \\
b_{1} & b_{2} & b_{3}
\end{array}\right|
\end{aligned}
$$

In the proof we want to show how and when, in general on $\mathrm{R}^{\mathrm{n}}$, we can construct a table multiplication of the orthonormal base, provided that the vector product thus defined has all the properties we require. Let us first generalize the definition of the vector product.

\section{Definition 2.1}

The vector product on $\mathrm{R}^{\mathrm{n}}$ is a binary operation $x: R^{n} \times R^{n} \rightarrow R^{n}$ which satisfies the properties of verticality, bilinearity and pytharorean property:

(i) $a \cdot(a \times b)=0$ and $b \cdot(a \times b)=0$, (verticality)

(ii) $(a \times b) \cdot(a \times b)+(a \cdot b)^{2}=(a \cdot a)(b \cdot b)$, (Pythagorean property)

(iii) $(\alpha \mathrm{a}+\beta \mathrm{b}) \times(\gamma \mathrm{c}+\delta \mathrm{d})=\alpha \gamma(a \times c)+$ $\alpha \delta(a \times d)+\beta \gamma(b \times c)+\beta \delta(b \times d)$ (Bilinearity)

The following properties follow from the definition of the vector product at $\mathrm{R}^{\mathrm{n}}$.

\section{Lemma 2.2}

Let $\mathrm{a}, \mathrm{b}$ and $\mathrm{c}$ be vectors from $\mathrm{R}^{\mathrm{n}}$. If the vector product on $\mathrm{R}^{\mathrm{n}}$ exists, then they are valid the following properties:

(2.1) $a \cdot(b \times c)=-b \cdot(a \times c)$;

(2.2) $a \times b=-b \times a$, which predominates $a \times$ $a=0$

(2.3) $a \times(a \times b)=(a \cdot b) a-(a . a) b$;

$$
\begin{aligned}
& (2.4) a \times(b \times c)=-((a \times b) \times c)+(c \cdot b) a+ \\
& (a \cdot b) c-2(a \cdot c) b
\end{aligned}
$$

Proof :

(2.1) From the perpendicularity of the vector product we conclude:

$$
\begin{aligned}
& 0=(b+a) \cdot((b+a) \times c) \\
& =(b+a) \cdot(b \times c+a \times c) \\
& =b \cdot(b \times c)+b \cdot(a \times c)+a \cdot(b \times c)+a \\
& \quad \cdot(a \times c) \\
& =b \cdot(a \times c)+a \cdot(b \times c) \\
& a \cdot(b \times c)=-b \cdot(a \times c) ;
\end{aligned}
$$

(2.2) We use the Pythagorean property of the vector product for the vector a:

$(a \times a) \cdot(a \times a)=(a \cdot a)^{2}-(a \cdot a)^{2}=0$

So it follows that $a \times a=0$. To prove the anticommutativity of the vector modulus we use bilinearity

$$
\begin{aligned}
& 0=(a+b) \times(a+b) \\
& =a \times a+a \times b+b \times a+b \times b \\
& =a \times b+b \times a
\end{aligned}
$$

So we get $a \times b=-b \times a$

(2.3) we now use the Pythagorean property for the vectors $(\mathrm{b}+\mathrm{c})$ and $\mathrm{a}$;

$((b+c) \times a) \cdot((b+c) \times a)$

$$
=((b+c) \cdot(b+c))(a \cdot a)-((b+c) \cdot a)^{2}
$$

Let's use bilinearity:

$$
\begin{aligned}
& ((b+c) \cdot(b+c))(a \cdot a)-((b+c) \cdot a)^{2}=(b \cdot b)(a \cdot a)+2(b \cdot c)(a \cdot a)+(c \cdot c)(a \cdot a)-(b \cdot a)^{2}- \\
& 2(b \cdot a)(c \cdot a)-(c \cdot a)^{2}
\end{aligned}
$$

In addition to the bilinearity of the vector and scalar product, and the Pythagorean property, we have:

$$
\begin{aligned}
& ((b+c) \times a) \cdot((b+c) \times a)=(b \times a+c \times a) \cdot(b \times a+c \times a) \\
& =(b \times a) \cdot(b \times a)+2(c \times a) \cdot(b \times a)+(c \times a) \cdot(c \times a) \\
& =(b \cdot b)(a \cdot a)-(b \cdot a)^{2}+2(c \times a) \cdot(b \times a)+(c \cdot c)(a \cdot a)-(c \cdot a)^{2}
\end{aligned}
$$


We equate (1) and (2) and then use (2.1) and (2.2). Then for all $\mathrm{c}$ is valid:

$$
\begin{aligned}
& c \cdot(a \times(a \times b))=-(b \times a) \cdot(c \times a) \\
& =(b \cdot a)(c \cdot a)-(b \cdot c)(a \cdot a) \\
& =c \cdot((b \cdot a) a-(a \cdot a) b)
\end{aligned}
$$

It follows that $a \times(a \times b)=(a \cdot b) a-(a . a) b$

(2.4) To prove the latter property, we again use the bilinearity of the vector product and properties (2.2) and (2.3)

$$
\begin{aligned}
& (a+c) \times((a+c) \times(b+c))=(a+c) \times(a \times b+a \times c+c \times b+c \times c)=a \times(a \times b)+a \times(a \times c)+ \\
& a \times(c \times b)+a \times(c \times c)+c \times(a \times b)+c \times(a \times c)+c \times(c \times b)+c \times(c \times c)=-a \times(b \times c)-c \times \\
& (b \times a)-(a \cdot a) b+(a \cdot b) a-(a \cdot a) c+(a \cdot c) a-(c \cdot c) b+(c \cdot b) c+(c \cdot c) a-(c \cdot a) c \quad \ldots \ldots \ldots \ldots(3)
\end{aligned}
$$

We now apply (2.3) to the vectors $(\mathrm{a}+\mathrm{c})$ and $(\mathrm{b}+\mathrm{c})$ :

$$
\begin{aligned}
& (a+c) \times((a+c) \times(b+c))=((a+c) \cdot(b+c))(a+c)-((a+c) \cdot(a+c))(b+c)=(a \cdot b+a \cdot c+ \\
& c \cdot b+c \cdot c) a+(a \cdot b-a \cdot c+c \cdot b-a \cdot a) c-(a \cdot a+2 a \cdot c+c \cdot c)
\end{aligned}
$$

Finally, by equating (3) and (4) we obtain $a \times(b \times c)=-((a \times b) \times c)+(c \cdot b) a+(a \cdot b) c-2(a \cdot c) b$

\section{Corollary 2.3}

Let $\mathrm{a}, \mathrm{b}$ and $\mathrm{c}$ be orthogonal unit vectors from $\mathrm{R}^{\mathrm{n}}$. if the vector posses on $\mathrm{R}^{\mathrm{n}}$ exists, then it must have the following properties:

$$
\begin{aligned}
& \text { (2.5) } a \times(a \times b)=-b ; \\
& \text { (2.6) } a \times(b \times c)=-((a \times b) \times c)
\end{aligned}
$$

Proof: The proof follows from the previous lemma

$$
\begin{aligned}
& (2.5) a \times(a \times b)=(a \cdot b) a-(a \cdot a)=-b \\
& (2.6) a \times(b \times c)=-((a \times b) \times c+(c \cdot b) a+(a \cdot b) c-2(a \cdot c) b=-((a \times b) \times c)
\end{aligned}
$$

In both cases we used the orthogonality of the vector, i.e. $a \cdot b=a \cdot c=b \cdot c=0$

We have now defined all the basic properties of the vector product we are looking for. Next the step is to construct a vector multiplication table for the orthornormal base at $\mathrm{R}^{\mathrm{n}}$. We denote the unit vector in $\mathrm{R}^{\mathrm{n}}$. we notice that in $\mathrm{R}^{3}$ we have $\left\{\mathrm{e}_{1}, \mathrm{e}_{2}, \mathrm{e}_{3}\right\}=\left\{\mathrm{e}_{1}, \mathrm{e}_{2}, \mathrm{e}_{1} \times \mathrm{e}_{2}\right\}$. Let's see how we can generalize that idea. Let's define a series of sets $\mathrm{S}_{\mathrm{k}}$ with:

$$
\begin{aligned}
& \mathrm{S}_{0}=\left\{\mathrm{u}_{0}\right\} \\
& \mathrm{S}_{\mathrm{k}}=\mathrm{S}_{\mathrm{k}-1} \cup\left\{\mathrm{u}_{\mathrm{k}}\right\} \cup\left(\mathrm{S}_{\mathrm{k}-1} \times \mathrm{u}_{\mathrm{k}}\right), \text { where } \mathrm{u}_{\mathrm{k}} \perp \mathrm{S}_{\mathrm{k}-1} \text {, that is, it is valid: } \\
& u_{k} \cdot u=0, \text { for all } \mathrm{u} \in \mathrm{S}_{\mathrm{k}-1}
\end{aligned}
$$

Consider the sets $\mathrm{S}_{0}, \mathrm{~S}_{1}, \mathrm{~S}_{2}$ and $\mathrm{S}_{3}$;

$$
\begin{aligned}
& \mathrm{S}_{0}=\left\{\mathrm{u}_{0}\right\} \\
& S_{1}=S_{0} \cup\left\{u_{1}\right) \cup\left(S_{0} \times u_{1}\right)=\left\{u_{0}, u_{1}, u_{0} \times u_{1}\right\} ; \\
& S_{2}=S_{1} \cup\left\{u_{2}\right) \cup\left(S_{1} \times u_{2}\right)=\left\{u_{0}, u_{1}, u_{0} \times u_{1}, u_{2}, u_{0} \times u_{2}, u_{1} \times u_{2},\left(u_{0} \times u_{1}\right) \times u_{2}\right\} ; \\
& S_{3}=S_{2} \cup\left\{u_{3}\right) \cup\left(S_{2} \times u_{3}\right) \\
& =\left\{u_{0}, u_{1}, u_{0} \times u_{1}, u_{2}, u_{0} \times u_{2}, u_{1} \times u_{2},\left(u_{0} \times u_{1}\right) \times u_{2}, u_{3}, u_{0} \times u_{3}, u_{1} \times u_{3},\left(u_{0} \times u_{1}\right)\right. \\
& \left.\quad \times u_{3}, u_{2} \times u_{3},\left(u_{0} \times u_{2}\right) \times u_{3},\left(u_{1} \times u_{2}\right) \times u_{3},\left(\left(u_{0} \times u_{1}\right) \times u_{2}\right) \times u_{3}\right\}
\end{aligned}
$$

Note that $\mathrm{S}_{1}$ structurally corresponds to the set $\left\{\mathrm{e}_{1}, \mathrm{e}_{2}, \mathrm{e}_{1} \times \mathrm{e}_{2}\right\}$. Let us also define the vector product of the sets $\mathrm{S}_{\mathrm{k}}$

$$
S_{i} \times S_{j}=\left(u \times v ; u \in S_{i}, v \in S_{j}\right)
$$

And expensive

$$
\pm S_{i}=S_{i} \cup\left(-S_{i}\right)
$$

With the next two lemma we will show that the sets $S_{n}$ are othonormal and closed with respect to the vector product.

\section{Lemma 2.4.}

$S_{1}=\left\{u_{0}, u_{1}, u_{0} \times u_{1}\right\}$ is an orthonormal set. Further, $S_{1} \times S_{1}= \pm S_{1}$ 
Proof: Orthogonality follows from the definition of the vector product and the definition of the set $S_{1}$ :

$$
\begin{gathered}
u_{0} \cdot\left(u_{0} \times u_{1}\right)=0, \\
u_{1} \cdot\left(u_{0} \times u_{1}\right)=0, \\
u_{0} \cdot u_{1}=0 ;
\end{gathered}
$$

And the standardization from the definition of $S_{1}$ and properties (2.1),(2.2),(2.3):

$$
\begin{aligned}
& u_{0} \cdot u_{0}=u_{1} \cdot u_{1}=1, \\
& \left(u_{0} \times u_{1}\right) \cdot\left(u_{0} \times u_{1}\right)=u_{0} \cdot\left(u_{1} \times\left(u_{0} \times u_{1}\right)\right)=u_{0} \cdot u_{0}=1
\end{aligned}
$$

We also show that $S_{1} \times S_{1}= \pm S_{1}$. By (2.2) and (2.5) we have:

$$
\begin{gathered}
u_{1} \times\left(u_{0} \times u_{1}\right)=u_{0} \in \pm S_{1} \\
u_{0} \times\left(u_{0} \times u_{1}\right)=-u_{1} \in \pm S_{1}
\end{gathered}
$$

\section{Lemma 2.5}

$\mathrm{S}_{\mathrm{k}}$ is an orthonormal set. Further, $S_{k} \times S_{k}= \pm S_{k}$ and $\left|S_{k}\right|=2^{k+1}-1$

Proof: We prove by induction. For $\mathrm{k}=1$, the statement holds for the pervious lemma. Assumption we see that $\mathrm{S}_{\mathrm{k}-1}$ is an orthonormal set, and that $S_{k-1} \times S_{k-1}= \pm S_{k-1}$ and $\left|S_{k-1}\right|=2^{k}-1$. We show that the statement holds for $\mathrm{S}_{\mathrm{k}}$. let $\mathrm{b}_{1}, \mathrm{~b}_{2}$ $\in S_{k-1}$. By definition, each element from $\mathrm{S}_{\mathrm{k}}$ form $\mathrm{b}_{1}, \mathrm{u}_{\mathrm{k}}$ or $\mathrm{b}_{1} \times \mathrm{u}_{\mathrm{k}}$. From (2.1),(2.2) and (2.5) it follows:

$$
\begin{aligned}
& b_{1} \cdot\left(b_{2} \times u_{k}\right)=u_{k} \cdot\left(b_{1} \times b_{2}\right)=0 \text { due to } u_{k} \perp S_{k-1} \text { and } b_{1} \times b_{2} \in S_{k-1} \\
& \text { And }\left(b_{1} \times u_{k}\right) \times\left(b_{2} \times u_{k}\right)=u_{k} \cdot\left(\left(u_{k} \times b_{1}\right) \times b_{2}\right)=-u_{k} \cdot\left(u_{k} \times\left(b_{1} \times b_{2}\right)\right)=0,
\end{aligned}
$$

So we conclude that $S_{k}$ is an orthorgonal set. Assuming induction, all element from $S_{k-1}$ are standardized, $u_{k}$ is standardized by definition and by $(2.1)$ and $(2.5)$ for $\mathrm{b}_{1} \times \mathrm{u}_{\mathrm{k}}$ is valid:

$$
\left(b_{1} \times u_{k}\right) \cdot\left(b_{1} \times u_{k}\right)=b_{1} \cdot\left(u_{k} \times\left(b_{1} \times u_{k}\right)\right)=b_{1} \cdot b_{1}=1
$$

Thus, it is true that $S_{\mathrm{k}}$ is also normalized. To prove that $S_{k} \times S_{k}= \pm S_{k}$ is valid, let us remember that $\mathrm{S}_{\mathrm{k}}=\mathrm{S}_{\mathrm{k}-1}$ $\cup\left\{u_{k}\right\} \cup\left(S_{k-1} \times u_{k}\right)$ and $\pm S_{k}=S_{k} \cup\left(-S_{k}\right)$. By the assumption of induction $S_{k-1} \times S_{k-1}$ is $S_{k-1} \cup\left(-S_{k-1}\right) \subseteq \pm S_{k}$, by definition it is $S_{k-1} \times u_{k} \subseteq \pm S_{k}, u_{k} \times\left(b_{1} \times u_{k}\right)=b_{1} \in S_{k-1} \subseteq \pm S_{k}$. It remains to show the following:

$$
\begin{gathered}
b_{1} \times\left(b_{2} \times u_{k}\right)=-\left(\left(b_{1} \times b_{2}\right) \times u_{k}\right) \in \pm S_{k} \text { and } b_{1} \times\left(b_{1} \times u_{k}\right)=-u_{k} \in \pm S_{k}, \\
\left(b_{1} \times u_{k}\right) \times\left(b_{2} \times u_{k}\right)=-b_{1} \times\left(u_{k} \times\left(b_{2} \times u_{k}\right)\right)=-=-b_{1} \times b_{2} \in \pm S_{k}
\end{gathered}
$$

It follows that $S_{\mathrm{k}}$ is an orthonormal set, $S_{k} \times S_{k}= \pm S_{k}$ and $2\left|S_{k-1}\right|+1=2\left(2^{k}-1\right)+2=2^{k+1}-1$

Lemma 2.5 shows us how to construct a multiplication table for a vector product. Thus due to bilinearity, a vector product can be defined on $\mathrm{R}^{\mathrm{n}}$ only if its $\mathrm{S}_{\mathrm{k}}$ base is for some $\mathrm{k}$. it is now clear that in this case $\mathrm{n}=\left|S_{k}\right|$ is valid i.e. $\mathrm{n}=2^{k+1}-1$

For $\mathrm{k}=1$, we observe $\mathrm{R}^{3}$. The multiplication table corresponds to the construction explained in lemma 2.5, with $\mathrm{e}_{1}=\mathrm{u}_{0}, \mathrm{e}_{2}=\mathrm{u}_{1}$ and $\mathrm{e}_{3}=\mathrm{u}_{0} \times \mathrm{u}_{1}$. Let's see what the table for $\mathrm{k}=2$ would look like, with the corresponding base $\left\{\mathrm{e}_{1}, \mathrm{e}_{2}, \ldots, \mathrm{e}_{7}\right\}$ for $\mathrm{R}^{7}$. With the help of lemma 2.5 , we generate a database:

$$
\begin{array}{llll}
e_{1}=u_{0} ; & e_{2}=u_{1} ; & e_{3}=u_{0} \times u_{1} ; & e_{4}=u_{2} ; \\
e_{5}=u_{0} \times u_{2} ; & e_{6}=u_{1} \times u_{2} ; & e_{7}=\left(u_{0} \times u_{1}\right) \times u_{2} &
\end{array}
$$

And then using properties (2.2), (2.5) and (2.6) we calculate the elements of the vector multiplication table:

$$
\begin{aligned}
& e_{i} \times e_{i}=0, i=1, \ldots \ldots 7 ; \\
& e_{1} \times e_{2}=u_{0} \times u_{1}=e_{3} ; \\
& e_{1} \times e_{3}=u_{0} \times\left(u_{0} \times u_{1}\right)=-u_{1}=-e_{2} ; \\
& e_{1} \times e_{4}=u_{0} \times u_{2}=e_{5} ; \\
& e_{1} \times e_{5}=u_{0} \times\left(u_{0} \times u_{2}\right)=-u_{2}=-e_{4} ; \\
& e_{1} \times e_{6}=u_{0} \times\left(u_{1} \times u_{2}\right)=-\left(u_{0} \times u_{1}\right) \times u_{2}=-e_{7} ; \\
& e_{1} \times e_{7}=u_{0} \times\left(\left(u_{0} \times u_{1}\right) \times u_{2}\right)=-\left(u_{0} \times\left(u_{0} \times u_{1}\right)\right) \times u_{2}=u_{1} \times u_{2}=e_{6} ; \\
& e_{2} \times e_{3}=u_{1} \times\left(u_{0} \times u_{1}\right)=u_{0}=e_{1} ; \\
& e_{2} \times e_{4}=u_{1} \times u_{2}=e_{6} ; \\
& e_{2} \times e_{5}=u_{1} \times\left(u_{0} \times u_{2}\right)=\left(u_{0} \times u_{1}\right) \times u_{2}=e_{7} ; \\
& e_{2} \times e_{6}=u_{1} \times\left(u_{1} \times u_{2}\right)=-u_{2}=-e_{4} ; \\
& e_{2} \times e_{7}=u_{1} \times\left(\left(u_{0} \times u_{1}\right) \times u_{2}\right)=-\left(u_{1} \times\left(u_{0} \times u_{1}\right)\right) \times u_{2}=-u_{0} \times u_{2}=-e_{5} ; \\
& e_{3} \times e_{4}=\left(u_{0} \times u_{1}\right) \times u_{2}=e_{7} ;
\end{aligned}
$$




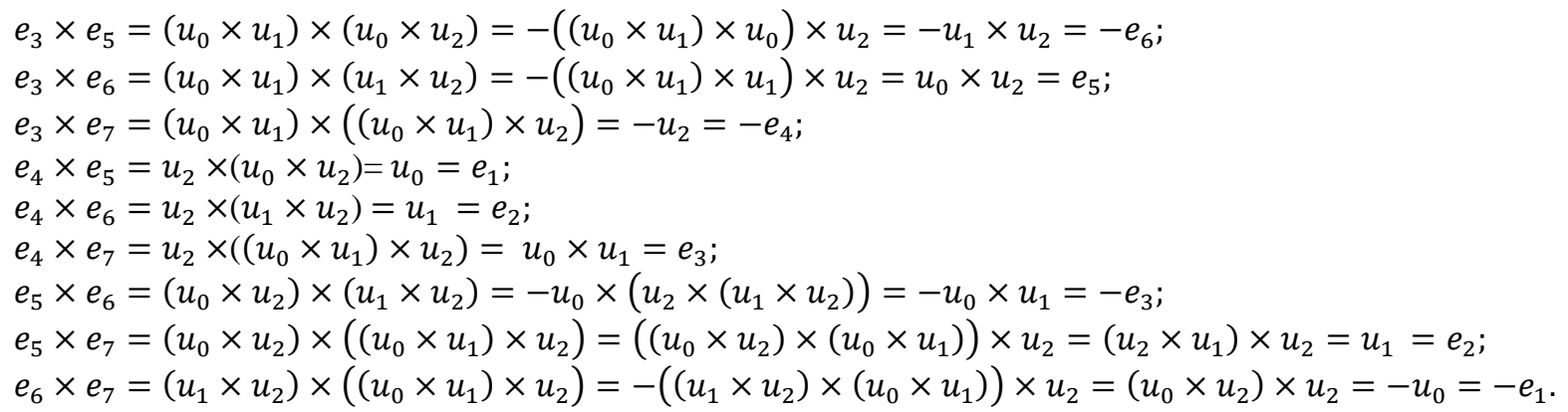

For elements below the diagonal, we use property (2.2) i.e the anticommutativity vector product. Thus, the multiplication table for $\mathrm{R}^{7}$ is as follows:

\begin{tabular}{|c|c|c|c|c|c|c|c|}
\hline$\times$ & $e_{1}$ & $e_{2}$ & $e_{3}$ & $e_{4}$ & $e_{5}$ & $e_{6}$ & $e_{7}$ \\
\hline$e_{1}$ & 0 & $e_{3}$ & $-e_{2}$ & $e_{5}$ & $-e_{4}$ & $-e_{7}$ & $e_{6}$ \\
\hline$e_{2}=$ & $-e_{3}$ & 0 & $e_{1}$ & $e_{6}$ & $e_{7}$ & $-e_{4}$ & $-e_{5}$ \\
\hline$e_{3}$ & $e_{2}$ & $-e_{1}$ & 0 & $e_{7}$ & $-e_{6}$ & $e_{5}$ & $-e_{4}$ \\
\hline$e_{4}$ & $-e_{5}$ & $-e_{6}$ & $-e_{7}$ & 0 & $e_{1}$ & $e_{2}$ & $e_{3}$ \\
\hline$e_{5}$ & $e_{4}$ & $-e_{7}$ & $e_{6}$ & $-e_{1}$ & 0 & $-e_{3}$ & $e_{2}$ \\
\hline$e_{6}$ & $e_{7}$ & $e_{4}$ & $-e_{5}$ & $-e_{2}$ & $e_{3}$ & 0 & $-e_{1}$ \\
\hline$e_{7}$ & $-e_{6}$ & $e_{5}$ & $e_{4}$ & $-e_{3}$ & $-e_{2}$ & $e_{1}$ & 0 \\
\hline
\end{tabular}

Using the table and bilinearity of the vector product, we can express an explicit formulae for calculating the vector product in $\mathrm{R}^{7}$. For vectors $\mathrm{a}=\left(\mathrm{a}_{1}, \mathrm{a}_{2}, \mathrm{a}_{3}, \mathrm{a}_{4}, \mathrm{a}_{5}, \mathrm{a}_{6}, \mathrm{a}_{7}\right)$ and $\mathrm{b}=\left(\mathrm{b}_{1}, \mathrm{~b}_{2}, \mathrm{~b}_{3}, \mathrm{~b}_{4}, \mathrm{~b}_{5}, \mathrm{~b}_{6}, \mathrm{~b}_{7}\right)$ their vector product is equal to: $a \times b=\left(-a_{3} b_{2}+a_{2} b_{3}-a_{5} b_{4}+a_{4} b_{5}-a_{6} b_{7}+a_{7} b_{6}\right) e_{1}+\left(-a_{1} b_{3}+a_{3} b_{1}-a_{6} b_{4}+a_{4} b_{6}-a_{7} b_{5}+\right.$ $\left.a_{5} b_{7}\right) e_{2}+\left(-a_{2} b_{1}+a_{1} b_{2}-a_{7} b_{4}+a_{4} b_{7}-a_{5} b_{6}+a_{6} b_{5}\right) e_{3}+\left(-a_{1} b_{5}+a_{5} b_{1}-a_{2} b_{6}+a_{6} b_{2}-a_{3} b_{7}+\right.$ $\left.a_{7} b_{3}\right) e_{4}+\left(-a_{4} b_{1}+a_{1} b_{4}-a_{2} b_{7}+a_{7} b_{2}-a_{6} b_{3}+a_{3} b_{6}\right) e_{5}+\left(-a_{7} b_{1}+a_{1} b_{7}-a_{4} b_{2}+a_{2} b_{4}-a_{3} b_{5}+\right.$ $\left.a_{5} b_{3}\right) e_{6}+\left(-a_{5} b_{2}+a_{2} b_{5}-a_{4} b_{3}+a_{3} b_{4}-a_{1} b_{6}+a_{6} b_{1}\right) e_{7}$

\section{Theorem 2.6}

Formula (2.1) defines the vector product on $\mathrm{R}^{7}$.

Proof: It is proved by direct verification of properties (i), (ii) and (iii) and in definition 2.1) we skip the detail of the evidence due to technical complexity.
Note 2.7

The vector product on $\mathrm{R}^{7}$ doesn not satisfy jacobi's identiy, which is easy check from definition.

So far we have shown that if a vector product on $\mathrm{R}^{\mathrm{n}}$ exists then $\mathrm{n}=2^{k+1}-1$ and Lemma 2.5 tells us how to construct a multiplication table for computation product, while retaining all the properties from the definition.

\section{Lemma 2.8}

Let $\mathrm{u}=\mathrm{u}_{0} \times \mathrm{u}_{1}+\mathrm{u}_{1} \times \mathrm{u}_{3}$ and $\mathrm{v}=\mathrm{u}_{1} \times \mathrm{u}_{2}-\left(\left(\mathrm{u}_{0} \times \mathrm{u}_{1}\right) \times \mathrm{u}_{2}\right) \times \mathrm{u}_{3}$. Then it is $\mathrm{u} \times \mathrm{v}=0$ and $\mathrm{u} \perp \mathrm{v}$

Proof: We use (2.2),(2.5) and (2.6)

$\mathrm{u} \times \mathrm{v}=\left(\mathrm{u}_{0} \times \mathrm{u}_{1}+\mathrm{u}_{1} \times \mathrm{u}_{3}\right) \times\left(\mathrm{u}_{1} \times \mathrm{u}_{2}-\left(\left(\mathrm{u}_{0} \times \mathrm{u}_{1}\right) \times \mathrm{u}_{2}\right) \times \mathrm{u}_{3}\right)$

$=\left(u_{0} \times u_{1}\right) \times\left(u_{1} \times u_{2}\right)-\left(u_{0} \times u_{1}\right) \times\left(\left(\left(u_{0} \times u_{1}\right) \times u_{2}\right) \times u_{3}\right)+\left(u_{1} \times u_{3}\right) \times\left(u_{1} \times u_{2}\right)-\left(u_{1} \times u_{3}\right) \times\left(\left(\left(u_{0} \times u_{1}\right) \times u_{2}\right) \times u_{3}\right)$

$=u_{0} \times u_{2}-u_{2} \times u_{3}-u_{3} \times u_{2}-u_{0} \times u_{2}=0$

For the second part of the statement, we note that $\mathrm{u}_{0} \times \mathrm{u}_{1}, \mathrm{u}_{1} \times \mathrm{u}_{3}, \mathrm{u}_{1} \times \mathrm{u}_{2}$ and $\left(\left(\mathrm{u}_{0} \times \mathrm{u}_{1}\right) \times \mathrm{u}_{2}\right) \times \mathrm{u}_{3}$ respectively are elements of $S_{k}$, for $\mathrm{k}>2$, and by lemma 2.5 we know that they are mutually orthogonal.

\section{Lemma 2.9}

Let $=u_{0} \times u_{1}+u_{1} \times u_{3}$ and $v=u_{1} \times u_{2}-\left(\left(u_{0} \times u_{1}\right) \times u_{2}\right) \times u_{3}$. Then $(u \cdot u)(v \cdot v)=4 \neq 0=(u \times v) \cdot(u \times v)+(u \cdot v)^{2}$.

Proof: Vector $\mathrm{u}_{0} \times \mathrm{u}_{1}, \mathrm{u}_{1} \times \mathrm{u}_{3}, \mathrm{u}_{1} \times \mathrm{u}_{2}$ and $\left(\left(\mathrm{u}_{0} \times \mathrm{u}_{1}\right) \times \mathrm{u}_{2}\right) \times \mathrm{u}_{3}$ respectively are elements of $\mathrm{S}_{\mathrm{k}}$, for $\mathrm{k}>2$, So they are also orthonormal and are valid $u \cdot u=v \cdot v=2, u \cdot v=0$, and from the pervious lemma it is valid $u \times v=0$, what pulls $(\mathrm{u} \cdot \mathrm{u})(\mathrm{v} \cdot \mathrm{v})=4 \neq 0=(\mathrm{u} \times \mathrm{v}) \cdot(\mathrm{u} \times \mathrm{v})+(\mathrm{u} \cdot \mathrm{v})^{2}$ 
Theorem 2.10

The vector product at $\mathrm{R}^{\mathrm{n}}$ exists if and only if $\mathrm{n}=0,1,3$ or 7 . Furthermore, for $\mathrm{R}^{3}$ and $\mathrm{R}^{7}$ there are orthonormal bases $\mathrm{S}_{1}$ and $\mathrm{S}_{2}$ such that $S_{i} \times S_{i}=$ $\pm S_{i}, i=1,2$.

Proof: By lemma 2.5, we see that the vector product exists only on $\mathrm{n}=2^{k+1}-1$. Next lemma 2.8 and 2.9 tells us that if we define a vector product on $R^{2^{k+1}-1}$, Pythagoras property is not valid for $\mathrm{k}>2$. It follows that a vector product with properties of verticality, bilinearity and Pythagorean property can exist only on $\mathrm{R}^{0}, \mathrm{R}^{1}, \mathrm{R}^{3}$ and $\mathrm{R}^{7}$. Theorem 2.6 proves the existence of a vector product on $\mathrm{R}^{7}$. Furthermore, trivial mapping (which maps all pairs of vectors to zero vector) defines the vector product of $R^{0}$ and $R^{1}$. The construction of the vector product on $\mathrm{R}^{3}$ is already done in 1. Finally, lemma 2.5 tells us how to generate orthonormal bases $\mathrm{S}_{1}$ and $\mathrm{S}_{2}$ for $\mathrm{R}^{3}$ and $\mathrm{R}^{7}$, such that it is worth $S_{i} \times S_{i}= \pm S_{i}, i=1,2$.

\section{Application of Cross Product}

Cross product has many applications in mathematics, physics, engineering, and computer programming. Few conceptual applications are as explained.

\section{Angular Velocity}

The angular velocity at any point is equal to half of the curl of the linear velocity at that point of the body i.e.

$$
\vec{\Omega}=\frac{1}{2} \operatorname{curl} \vec{v}
$$

And curl may be defined as follows by cross product

Let $\vec{v}$ be a vector valued function, then curl of $\vec{v}$ is denoted by $\operatorname{curl} \vec{v}$ or $\nabla \times \vec{v}$ and is defined by $\nabla \times \vec{v}=\left(\vec{\imath} \frac{\partial}{\partial x}+\vec{\jmath} \frac{\partial}{\partial y}+\vec{k} \frac{\partial}{\partial z}\right) \times \vec{v}$

\section{Volume Integrals}

Any integral which is to be evaluated over a volume is called a volume integral. We have a use of cross product on volume integral as:

Let $\mathrm{S}$ be a piece wise smooth oriented surface in space and the boundary of $\mathrm{S}$ be a piecewise smooth simple closed curve C. Let $\vec{F}(\mathrm{x}, \mathrm{y}, \mathrm{z})$ be a continuos vector valued function that has continuous first partial derivates in a domain in space containing $\mathrm{S}$. Then $\iint_{S}(\operatorname{Curl} \vec{F} \cdot \vec{n}) d s=\oint_{C} \vec{F} \cdot d \vec{r}$, where $\vec{n}$ is a unit normal vector of $S$ and the integration around $C$ is taken in anticlockwise direction with respect of $\vec{n}$.

\section{Orbital Angular Momentum in 3 D}

In vector notation, the orbital angular momentum of a point particle in motion about the origin is defined as: $\mathrm{L}=\mathrm{I} \omega$
Where,

$\mathrm{I}=r^{2} \mathrm{~m}$ is the moment of inertia for a point mass $\omega=\frac{r x v}{r^{2}}$ is the orbital angular velocity in $\mathrm{rad} / \mathrm{sec}$ of the particle about the origin

$r$ is the position vector of the particle relative to the origin, and

$\mathrm{m}$ is the mass of the particle

This by the rules of vector algebra can be rearranged as:

$$
\begin{aligned}
& \mathrm{L}=\left(r^{2} \mathrm{~m}\right)\left(\frac{r x v}{r^{2}}\right) \\
& =\mathrm{m}(\mathrm{r} \times \mathrm{v}) \\
& =\mathrm{r} \times \mathrm{mv} \\
& =\mathrm{r} \times \mathrm{p}
\end{aligned}
$$

Which is the cross product of the position vector $r$ ad the linear momentum $p=m v$ of the particle. By the definition of the cross product, the $\mathrm{L}$ vector is perpendicular to both $\mathrm{r}$ and $\mathrm{p}$.

\section{Game Programming}

Knowledge of cross product is also useful to any programmer interested in 3D computer graphics or $3 \mathrm{D}$ computer game programming.

If we say the vectors $(i, j, k)(i, j, k)$ are the vectors that form the orthogonal basis for our coordinate space, then the cross product satisfies the following identities:

$$
\begin{aligned}
& \mathrm{i} \times \mathrm{j}=\mathrm{k} \\
& \mathrm{j} \times \mathrm{k}=\mathrm{i} \\
& \mathrm{k} \times \mathrm{i}=\mathrm{j}
\end{aligned}
$$

And if we swap the order of the vectors for the cross product, the result will be negated, i.e. to say the result vector will have opposite direction.

$$
\begin{aligned}
& \mathrm{j} \times \mathrm{i}=-\mathrm{k} \\
& \mathrm{k} \times \mathrm{j}=-\mathrm{i} \\
& \mathrm{i} \times \mathrm{k}=-\mathrm{j}
\end{aligned}
$$

It is also useful to note, that when switching from a left-handed coordinate system and a righthanded coordinate system, one of the axes is inverted (traditionally the z-axis is inverted). This means that if in a left-handed coordinate system the result of the cross product on the unit basis vectors $(\mathrm{x}, \mathrm{y}, \mathrm{z})$ :

$$
\mathrm{x} \times \mathrm{y}=\mathrm{z}
$$

This means that in a left-handed coordinate system, the result of the cross product will point away from the viewer (into the screen), but in a right-handed coordinate system, the result of the same cross product will point towards the viewer (out of the screen). This is a by-product of the handedness of the coordinate system. More clearly, right-handedness signifies any positive axis $(\mathrm{x}, \mathrm{y}$, or $\mathrm{z})$ pointing towards the viewer whereas; left-handedness signifies any positive axis ( $\mathrm{x}$, $\mathrm{y}$, or $\mathrm{z}$ ) pointing away from the viewer. Hence cross product is an essential part in graphics programming. 


\section{Computational Geometry}

Computational geometry is a branch of computer science which deals with the study of algorithms that aims at solving problems about geometric objects. Its application areas include computer graphics, computer-aided design and geographic information systems, robotics, and so on.

The cross product appears in the calculation of the distance of two skew lines which are not in the same plane from each other in $3 \mathrm{D}$ space. The cross product is also extensively used to calculate the normal for a triangle or polygon which is an operation often used in computer graphics. For example, the winding of a polygon either clockwise or anticlockwise about a point within the polygon can be calculated by triangulating the polygon (like spokes of a wheel) and summing the angles between the spokes by using the cross product to keep a pathway of the sign of each angle. Besides, the cross product is used in calculating the volume of a polyhedron such as a tetrahedron or parallelepiped.

\section{CONCLUSION}

Given the properties of bilinearity, orthogonality and magnitude, a nonzero cross product exists only in three and seven dimensions. This can be shown by postulating the properties required for the cross product, then deducing an equation which is only satisfied when the dimension is $0,1,3$ or 7 . In zero dimensions there is only zero vector, while in one dimension all vectors are parallel, So in both these cases the product must be identically zero. Moreover, we have shown the existence of cross product on $\mathrm{R}^{\mathrm{n}}$ starting from the definition and properties of cross product.

\section{ACKNOWLEDGEMENTS}

I owe and highly indebted to Mrs. Sumintra Kumari Majhi for her generous support during the preparation of the paper. Especial thanks to Er. Binod Aryal for his constructive suggestion and inspiration throughout this research work. We want to dedicate it to medical professional for saving us, keeping them at risk.

\section{REFERENCES}

1. Alberto Elduque, Vector Cross Products, Electronic copy found at: http://www.unizar.es/matematicas/algebra/elduque /Talks/crossproducts.pdf

2. Adams, J. F. (1960). On the non existence of elements of Hopf invariant one, Ann. Of Math., 72, 20-204.

3. Postnikov, M. (1986). Lectures in Geometry: Lie groups and Lie algebras, Moscow.

4. Matea, P. (2015). Vector Product on $R^{n}$, thesis.

5. Mcloughlin, P. F. When does a cross product on $R^{n}$ exist?, arXiv:1212.3515.

6. Mcloughlin, P. F. Basic Properties of Cross Products. Electronic copy found at: http://www. math. csusb. edu/faculty/pmclough/BP. pdf.

7. Toya, N. P. (2013). Enginerring Mathematics-III.

8. Massey, W. S. (1983). Cross Products of Vectors in Higher Dimensional Euclidean Spaces, The American Mathematical Monthly, 90(10), $697-$ 701. 